\title{
Globe
}

Revue internationale d'études québécoises

\section{Louise H. Forsyth [éd.] : Nicole Brossard. Essays on Her Works. Toronto/Buffalo/Chicago/Lancaster (U.K.), Guernica, 2005}

\section{Denisa Oprea}

Volume 9, numéro 1, 2006

URI : https://id.erudit.org/iderudit/1000810ar

DOI : https://doi.org/10.7202/1000810ar

Aller au sommaire du numéro

Éditeur(s)

Globe, Revue internationale d'études québécoises

ISSN

1481-5869 (imprimé)

1923-8231 (numérique)

Découvrir la revue

Citer ce compte rendu

Oprea, D. (2006). Compte rendu de [Louise H. Forsyth [éd.] : Nicole Brossard. Essays on Her Works. Toronto/Buffalo/Chicago/Lancaster (U.K.), Guernica, 2005]. Globe, 9(1), 292-296. https://doi.org/10.7202/1000810ar d'utilisation que vous pouvez consulter en ligne.

https://apropos.erudit.org/fr/usagers/politique-dutilisation/ 


\section{Louise H. Forsyth [éd.] \\ Nicole Brossard. Essays on Her Works. Toronto/Buffalo/Chicago/Lancaster (U.K.),} Guernica, 2005.

Poète et romancière, intellectuelle de marque et figure de proue du féminisme québécois, Nicole Brossard signe une œuvre qui a influencé de façon décisive l'évolution de la littérature québécoise des quarante dernières années. Riche et abondante, sa création est aussi largement commentée. Le véritable foisonnement métatextuel qu'elle engendre s'inscrit souvent dans une vulgate critique qui s'articule autour des mêmes lieux communs. Cela pose, pour l'exégète contemporain, le défi du renouveau du discours critique.

Ce défi, les essais réunis dans Nicole Brossard. Essays on Her Works le relèvent de manière convaincante. À la fois denses et rigoureux, puisant à des approches différentes, ils évitent les poncifs d'une œuvre abondamment interprétée et l'éclairent sous des angles nouveaux. Ils en confirment par le fait même l'exceptionnelle fécondité et pérennité.

D'emblée, la formule éditoriale proposée par Louise $H$. Forsyth transgresse la logique consacrée d'un simple recueil d'essais. Des poèmes inédits, rassemblés sous le titre "Shadow, Soft et soif ", et des "Fragments of a Conversation " avec Nicole Brossard précèdent les dix études proprement dites. L'éditrice signe également un essai introductif, "To Write: In the Feminine Is Heavy with Consequences", remarquable par la clarté et la consistance des propos critiques, de même que par l'exemplaire connaissance - on pourrait même parler de connivence de l'œuvre de Brossard.

Sur un mode serein, lumineux, les poèmes réactivent des thèmes et des images récurrents de l'univers poétique brossardien: les mots, le silence, le désir, l'aube... Tout en posant que "vivre est nécessairement un atout inside language" (p. 11), ils renforcent une constante majeure du projet créateur de l'écrivaine. En effet, comme elle l'affirme aussi dans "Fragments of a Conversation", Brossard fait des mots, du langage, de l'écriture en général un moyen privilégié d'agir sur le monde, de le comprendre, de le réinventer. Dans ce processus, son statut de femme 
est déterminant. Son œu'vre se donne comme un effort continu, accompli dans et à travers le langage, pour remettre en question les structures mêmes - voire les structures patriarcales de la pensée, du savoir, de la mémoire, des passions - afin d'y inscrire, de dire le réel des femmes.

Ce sont justement les multiples facettes de cette entreprise, doublement ancrée dans l'idéologique/l'éthique et dans l'exploration/ l'invention formelle, que mettent en lumière toutes les essayistes. Deux axes principaux sous-tendent de ce point de vue chaque intervention et assurent la cohérence de l'ensemble. De prime abord, il s'agit de repérer certaines des stratégies textuelles, discursives et de représentation qui, tout en rejetant l'ordre établi des discours, permettent de faire advenir dans la parole l'inédit, le "untbought and unknown. (p. 209). Les études portent principalement sur le métissage générique, sur l'hologramme, sur les pratiques "délirantes " de lecture et de traduction, ou encore sur l'installation ou le travail de fictionnalisation - pour ne nommer ici que certains des procédés les plus typiquement brossardiens. Elles soulignent la préoccupation constante de l'écrivaine d'innover au cœur même des normes et des conventions littéraires. D'où la mise en relief, dans un deuxième temps, d'une dimension performative de l'écriture brossardienne qui, comme l'affirme Karen S. McPherson, "consciously explores the transformative possibilities of women's engagement with bistory and story/[Language $]^{7}$.

S'ils renvoient aussi, dans un but comparatif, à d'autres textes brossardiens, les quatre premiers essaiś abordent plus spécifiquement $\mathrm{Hier}$, le roman paru en 2001. À partir des images dominantes de la perte et de la mort, Karen S. McPherson ("Since Yesterday: Nicole Brossard's Writing after Loss") et Alice A. Parker ("Performativity in Hier ") posent l'écriture comme travail de deuil, où se construit une mémoire permettant à l'avenir d'exister. Écrire la mort, c'est guérir, sauver. On constate de ce point de vue un changement de visée dans le parcours éthique de l'écrivaine. Si elle se proposait, dans les romans antérieurs, de changer le cours de l'histoire, voire le cours de la mort, Brossard se donne ici le but moins ambitieux, plus intime, d'، irriguer là où le cœur est sec et demandant ". Car, comme le constate Louise Dupré dans l'un des essais les plus limpides et les plus convaincants du volume, en cette époque

7. " [E]xplore consciemment les possibilités de changement qu'entraîne l'action des femmes dans l'histoire et dans le récit/le langage [je traduis] ", p. 55. 


\section{REVUE INTERNATIONALE D'ÉTUDES QUÉBÉCOISES}

de fin des métarécits, l'écrivain ne saurait plus ambitionner de changer radicalement le monde. Il peut tout au plus se poser en témoin lucide de son temps, tout en veillant, commẹ le fait Brossard, à ce que la fiction agisse sur le réel. De ce point de vue, Hier est, selon Parker, "perhaps the most millennial of Brossard's texts ${ }^{8}$. Il interroge le sens de notre présence au monde, notre situation par rapport à un présent stérile, envahissant, de même que les possibles réponses inscrites au cœur de la modernité. Il affirme l'urgence d'inventer de nouveaux espaces mentaux et textuels où, pour reprendre la formule de Michel Foucault, il serait enfin possible de penser, voire de penser autrement. Entre ses pages, l'acte de langage se transforme nécessairement en un "conscious [act] of re-signification" (p. 82). Il réécrit l'histoire et la mort, réinvente le temps, la mémoire et l'amour, redéfinit le sujet, le pouvoir, le faire, le politique. Figures rhétoriques avec la consistance de la voix énonciative à l'œuvre dans la prose poétique, les personnages féminins deviennent en ce sens des archétypes de la femme contemporaine, "new symbols, modern myths" (p. 91).

Hautement impur, résistant à la totalisation, Hier s'attaque aussi aux canons littéraires. Il remet en question les notions traditionnelles de personnage, de linéarité, de causalité, de pureté générique. Il flirte, comme le démontre pertinemment Claudine Potvin dans "Flirting with the Museum Narrative ", avec les modalités narratives consacrées dans le but de mieux les subvertir, tandis qu'il impose sa structure plurielle, construite sur les modes du déplacement, de la condensation, de la surdétermination.

Inspirés notamment de Picture Theory, les essais de Katharine Conley ("Moving into the Third Dimension. Nicole Brossard's Picture Theory") et de Susan Knutson ("Feminist Legends of Natureculture. Brossard, Haraway, Science ") abordent les multiples aspects et fonctions de l'hologramme. Ce dernier oppose à la raison cartésienne sous-tendant l'édifice du patriarcat sa pulsion déviante, sa philosophie sensuelle et sa virtualité métonymique. Selon Conley, le modèle holographique régirait le fonctionnement des pratiques d' réécriture - ou de réécriture - et de lecture à l'œuvre dans Picture Theory. Il détermine en ce sens l'architecture prismatique du texte, dont les sections se réfractent l'une l'autre dans un mouvement qui est à la fois tentation et refus de la totalisation.

8. • [P]eut-être le texte de Brossard le plus millénariste lje traduis] „, p. 71. 
Il définit la dynamique sensuelle, émotionnelle du dialogue inter et intratextuel mis en place par l'écrivaine. Il pose enfin, par sa structure ouverte, la géométrie participative et interactive de l'acte de lecture. Dans une tout autre direction, pour Knutson, l'hologramme est (à l'image de) la "femme intégrale ", à la fois idéal, utopie et "political trope * (p. 168) du féminisme radical. Brisant la logique binaire des équations traditionnelle $($ femme $=$ nature) et essentialiste (femme $=$ culture), la "femme intégrale "marque l'inscription de la femme dans la totalité harmonieuse de la $\cdot$ natureculture .

À partir du concept de délire - entendu, en tant que modalité discursive, comme déviation par rapport à la norme -, Susan Holbrook ("Delirious Translations in the Works of Nicole Brossard") met en lumière la dimension (re)créative et la portée subversive des pratiques de lecture et de traduction telles qu'elles sont définies dans Le désert mauve, ou bien dans Mauve et Character/Jeu de lettres. À cette fête/quête délirante inscrite au cour du langage s'oppose la tentation du silence, cet espace privilégié de la compréhension et de l'expérience avec lequel le sujet féminin entretient des relations problématiques. Catherine Campbell ("Our Last Chance for Silence ") examine de ce point de vue les contradictions et tensions engendrées dans l'œuvre de Brossard par l'exploration du potentiel signifiant du silence. Rejeté dans Le désert mauve au nom de l'urgence éprouvée par le personnage féminin de s'assigner une place dans le langage, il devient pour le sujet pleinement affirmé de Baroque d'aube le seul lieu de sens au milieu du chaos contemporain.

Les deux derniers essais offrent des analyses fort élaborées des stratégies textuelles et de représentation par lesquelles l'écriture brossardienne, tout en exhibant sa dimension expérimentale, parvient à redéfinir les bases mêmes de notre présence au monde. Selon Barbara Godard ("Life (in) Writing. Or a Writing-Machine for Producing the Subject "), l'écrivaine s'emploie notamment à libérer le sujet du carcan patriarcal de la logique binaire et métaphorique. Elle vise aussi à transformer sa dispersion tautologique, synonyme d'épuisement, à l'intérieur d'un langage devenu intransitif, en une possibilité de survie et de changement. Elle explore en ce sens le potentiel créateur et novateur de certains procédés et modes de représentation, tels la métonymie, le jeu avec la chair physique, typographique, du texte, la virtualité désirante de la fiction/fictionnalisation. Par leur géométrie mouvante et subversive, 


\section{REVUE INTERNATIONALE D'ÉTUDES QUÉBÉCOISES}

ces procédés renvoient à l'image d'un sujet "focus[ed] on processes, not bounded entities or fixed states" ".

Dans l'essai le plus étoffé du recueil ("The Inédit in Writing by Nicole Brossard. Breatbing the Skin of Language"), Lynette Hunter pose la dimension morale que recèle, dans l'œuvre de Brossard, la production de sens. Ce processus se définit essentiellement comme un travail d'articulation, dans et par le langage, de l'inédit, de ce qui a été occulté par les modes traditionnels de représentation. L'essayiste raffine les étapes d'un cheminement esthétique et idéologique qui non seulement ouvre un "space for the existence of the woman subject and ber desire "10 ", mais explore des bases nouvelles qui permettent au sujet installé de s'affirmer sur la scène sociale en tant qu'agent moral.

Par l'originalité et la diversité des approches, par l'équilibre entre effet de théorisation et travail avec le texte - plutôt que sur le texte -, les dix essais réunis par Louise $\mathrm{H}$. Forsyth innovent à l'intérieur même de la (jeune) critique au féminin. Ils parviennent ainsi à rendre compte de la complexité d'une œuvre ayant, selon la formule de l'éditrice, "transformed Canada's socio-cultural landscape ${ }^{11}$.

Denisa Oprea

Université Laval

9. "[C]entré sur/par des processus, plutôt [qu'un sujet en tant que] entité délimitée ou état stable lje traduis] ", p. 196.

10. * [E]space pour l'existence du sujet féminin et de son désir lje traduis] ", p. 222. 11. [T]ransformé le paysage socioculturel canadien [je traduis] ", p. 35. 